\title{
Associations of Perinatal Factors with Childhood Asthma and Allergies: A Cross-Sectional Study in Tianjin, China
}

\author{
Agnes Ellie ${ }^{1}$, Yuexia Sun ${ }^{1}$, Jing Hou ${ }^{1}$, Xiangrui Kong ${ }^{1}$, Pan Wang ${ }^{1}$, Qingnan Zhang ${ }^{1}$, and \\ Jan Sundell ${ }^{1}$ \\ ${ }^{1}$ Tianjin University
}

May 15, 2020

\begin{abstract}
ABSTRACT Background: Asthma, rhinitis, and eczema are becoming increasingly prevalent among children in China. Studies have shown that the perinatal period is critical and impacts children's health. However, research on the associations between perinatal factors and childhood allergic diseases in China are few. Objective: To evaluate the associations between the home factors (dampness/ humidity, parental smoking, contact with animals, and renovation) and childhood allergic diseases around the perinatal periods in China. Method: This work is part of the China Child Health and Home (CCHH) study and is an analysis of data from a cross-sectional questionnaire investigation in urban Tianjin and rural Cangzhou, China, from 2013 to 2014. A total of 7,366 randomly selected kindergarten and elementary school children age 0-8 years old were investigated. The questions used in the study were modeled according to the Dampness in Buildings and Health (DBH) study conducted in Sweden. Results: The prevalence of children's doctor-diagnosed asthma, rhinitis, and eczema were 4.1\%, 9.5\%, and 35.6\%, respectively. There were associations between the indexes of dampness/humidity and allergic diseases, with an odd ratio of up to 4.02 at a $95 \%$ confidence interval: (1.87-8.64), after adjustment. The other perinatal factors (parental smoking, contact with animals, and renovation) also showed positive associations with the allergic conditions. Conclusion: The environmental factors around the perinatal period show strong associations with the allergic conditions discussed in this study. Further studies are needed to identify the biologic background and probable mechanisms for these associations.
\end{abstract}

\section{Associations of Perinatal Factors with Childhood Asthma and Allergies:}

\section{A Cross-Sectional Study in Tianjin, China}

Agnes Siah Ellie, Yuexia Sun, Jing Hou, Xiangrui Kong, Pan Wang, Qingnan Zhang, Jan Sundell

Tianjin Key Laboratory of Indoor Air Environmental Quality Control, School of Environmental Science and Engineering, Tianjin University, No.135 Yaguan Road, Haihe Education Park, Tianjin, 300350, China

Running Title: Perinatal factors and childhood allergy

\section{Corresponding author:}

Yuexia Sun, PhD, Associate Professor

Address: No.135 Yaguan Road, Haihe Education Park, Tianjin, 300350, China;

Office phone: 022-85356721;

E-mail: yuexiasun@tju.edu.cn

Word count: 2,391 Table count: 4

Hosted file 
Manuscript main text.docx available at https://authorea.com/users/322498/articles/451449associations-of-perinatal-factors-with-childhood-asthma-and-allergies-a-cross-sectionalstudy-in-tianjin-china 